\title{
COMPUTER GENERATED END SHIMS FOR RECYCLER RING MAGNETS
}

\author{
C.N. Brown, G.W. Foster ${ }^{+}$, G. P. Jackson, J. T. Volk, FNAL*, Batavia, IL
}

\begin{abstract}
The procedure for automatically producing customized end shims for the Recycler Antiproton Storage Ring gradient magnets is described. Magnets were first measured with a harmonics probe. The observed field defects were then fed into a computer program (initially a spreadsheet) which generated the toolpath code for a numerically controlled milling machine which produced individually customized end shims that eliminated the measured defect. Field defects through decapole were corrected to an accuracy (limited by the reproducibility of the measurement apparatus) to a few $\times 10^{-5}$.
\end{abstract}

\section{INTRODUCTION}

The Fermilab Recycler Antiproton Storage Ring [1] requires approximately 400 permanent magnets with field defects of order $10^{-4}$ or better for the low multipoles. Under these circumstances the circulating aperture is expected to be limited by the physical aperture of the magnets $(5 \mathrm{~cm}$ magnet gap with $\sim 4.5 \mathrm{~cm} \times 8 \mathrm{~cm}$ clear aperture inside beam pipe) rather than the dynamic aperture due to magnetic field defects [2].

Most of the Recycler magnets are gradient dipoles with gap fields of approximately 1.5kGauss. Their "hybrid" design uses temperature-compensated permanent magnet material [3] to drive the magnet and steel pole tips to shape the field. The steel is very well behaved at this field level (high permeability and low hysteretic effects) and the magnetic field errors are almost exclusively due to geometric effects in the steel shape and magnet assembly.

Experience with the early prototypes indicated that the observed multipoles in the as-built magnets were near or at the tolerable limit: a few $\times 10^{-4}$ gradient error, sextupole through decapole errors of order $10^{-4}$, and smaller amplitudes for higher multipoles. (Multipoles were measured a probe radius of $2 \mathrm{~cm}$ and expressed at a reference radius equal to the magnet half-gap of $2.54 \mathrm{~cm}$ ). Therefore to ensure that the field quality specifications were met, a procedure of applying an individual end shim to each magnet was initiated. This procedure was capable of trimming normal multipoles through decapole and allowed the field quality specifications for both systematic and random multipole errors to be exceeded.

+ email: GWF@FNAL.GOV

* Operated by Universities Research Association Inc., under contract with the U.S. Department of Energy.

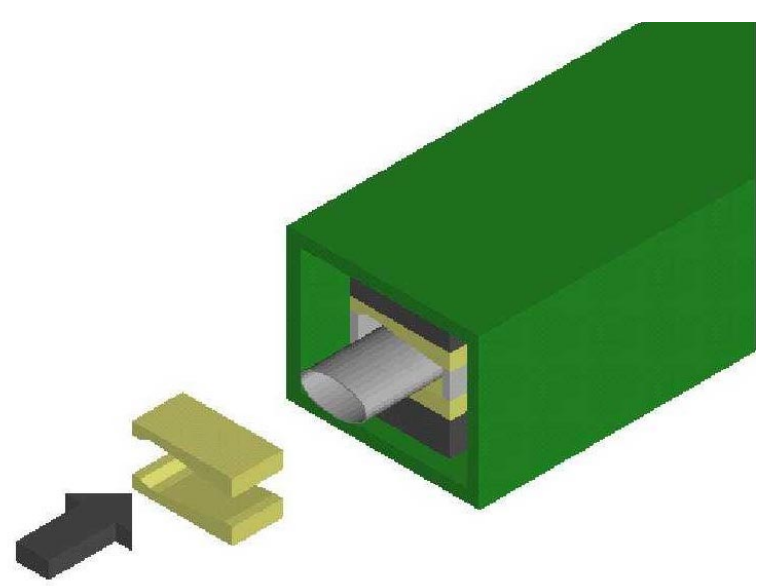

Fig. 1 - Recycler Permanent Magnets showing installation locations of shims on the ends of the gradient pole tips. The magnet ends were protected by steel cover plates (not shown).

\section{SHIMMING PROCEDURE}

The shimming procedure which evolved over the production of the magnets worked as follows:

1) Each assembled magnet was tested with a harmonics probe (a Morgan coil) which measured the low harmonics with an RMS accuracy of $1-2 \times 10^{-5}$.

2) The measurements were used to determine the shape of a custom piece of steel (a "Z-shim") which was applied to the ends of the pole tip. These were pieces of pole tip steel with the standard transverse cross section but cut to a variety of shapes as viewed from the top (see fig. 2).

3) The calculated shape was used to generate the toolpath programming for a numerically controlled (NC) vertical mill which machined the required piece from blank pole tip stock. Initially the toolpath code [4] was written out directly from a spreadsheet program, and later generated automatically by a subroutine called from the magnet analysis code. The steel shims were typically produced in batches at the FNAL machine shop.

4) The custom shim was applied to the magnet. This fiveminute procedure required a wrench and a little bit of experience in wrestling any previously mounted endshim off of the end of the energized permanent magnet.

5) The magnet was re-measured, and in occasional cases where the field quality still did not meet specifications, steps 2-5 were iterated. 
For many of the later series of magnets an inventory of "semi-custom" end shims was maintained which allowed the most common field defects to be cured with a prefabricated end shim. When it was possible to use one of the pre-built shims on a magnet it represented a considerable time savings since the magnet did not have to be dismounted and remounted on the magnet test stand while a custom shim was being machined.

It was decided to place an individualized shim on only one end of each magnet. The shim on the other end was a "standard" shim (identical for all magnets of a given type) which was chosen to correct for one-half of the average systematic defect as measured in the first 20 magnets of the production run. Having a standard shim on one end of the magnet leaves open the possibility of retrofitting a new standard shim on all magnets of a given type if modifications to the field shape of the magnets are desired after machine commissioning.

\section{END SHIM DESIGN}

The basic mechanism by which a z-shim works is that the integrated field of a magnet is proportional to the length of the pole tip. Thus if the end of pole is sliced diagonally, the magnet is stronger on side of the aperture than the other. This introduces an effective gradient in the integrated field ("edge focussing"). Similarly, if the pole tip is cut with a parabolic shape a sextupole field is generated. A cubic profile introduces an octupole and so on. See fig. 2. Thus it is possible to compensate small defects in the body field of a magnet by making changes to the end shape of the pole tip.

A number of issues complicate this simple picture. Firstly, the simple polynomial shaping of the pole tips does not generate a simple result: for example, a cubic Zshim profile will generate not only an octupole term but also a gradient shift. Secondly, there are a variety of feeddown effects present in gradient magnets. For example, if a gradient magnet is cut diagonally it generates not only a gradient but also a sextupole term ("edge sextupole") since the amount of quadrupole seen by the beam depends on the position across the aperture. Analogous effects exist for higher multipoles. Finally, the Z-shim is inherently a 3-dimensional field shaping technique and effects such as the interaction of the pole tip ends with the flux return box cannot be neglected.

In practice we had magnets to build, so that the approach taken was to build a set of trial shims with the elementary monomial defects (linear, quadratic, cubic, etc.). The multipole shifts induced by each of these shims was measured, then a matrix was inverted to determine which linear combinations of polynomial defects will produce e.g. one unit of pure decapole change. This orthogonalization procedure was iterated by producing a second set of shims designed to produce a pure shift in each of the low multipoles. Linear combinations of these shapes can be superimposed to cancel arbitrary sets of errors in the low multipoles.

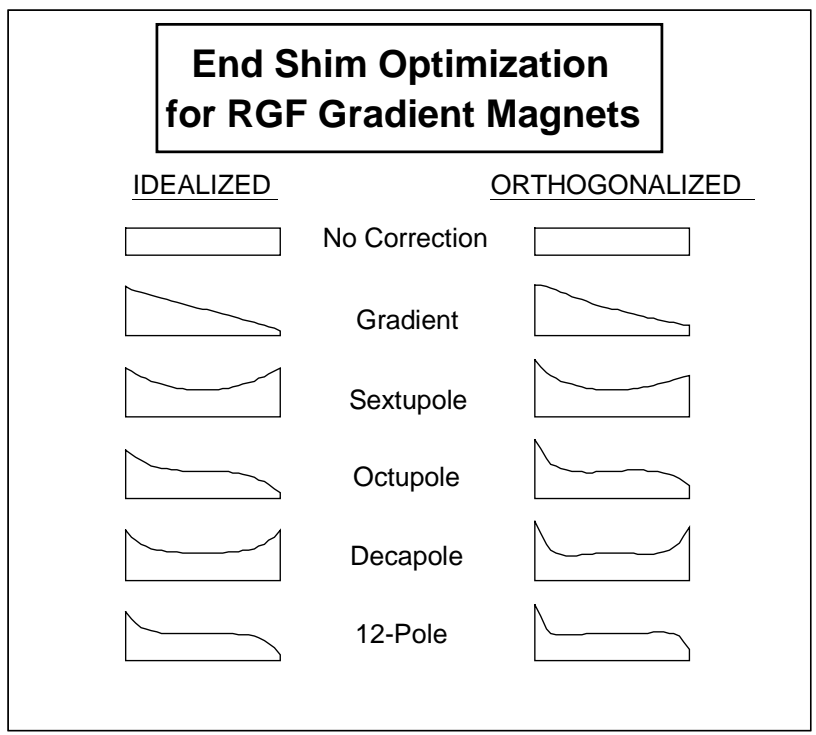

Fig. 2 - Elementary monomial Z-shim designs used as a starting point, and shim designs which were found to produce pure multipole shifts after re-orthogonalizing the multipole contributions from the elementary shims.

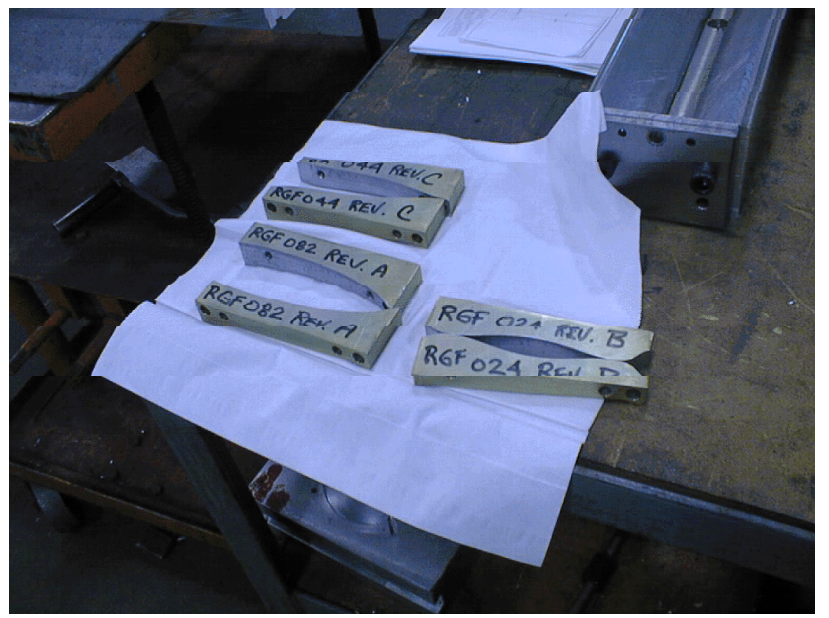

Figure 3: Customized end shims produced on a NC mill to null out error multipoles on Recycler magnets.

There is no guarantee that this process should be extremely linear. If a single correction term becomes large enough, it will produce a shim design that will extend the pole tip out near the flux return shield and behave very nonlinearly. Also, shim design which contains both quadrupole and decapole corrections may induce cross terms including, say, octupole. However in practice for the small $\left(\right.$ few $\times 10^{-4}$ ) defects being cancelled with the shims, they were adequately linear to achieve a 5-fold reduction in the error harmonics in a single iteration.

To get a feeling for the size of the end shimming needed, a gradient error of $10^{-4} / \mathrm{cm}$ in the body field of a 4 meter magnet can be compensated for by a z-shim slope of $(400 \mathrm{~cm}) * 10^{-4} / \mathrm{cm}=4 \%$. This corresponds to a slope of 
$6 \mathrm{~mm}$ across the width of a $15 \mathrm{~cm}$ pole tip. Shim corrections for higher multipoles get large fast, but errors in higher multipoles tend to fall off with multipole number just as fast. There was also a tendency for large corrections to be of opposite sign: for example a large positive sextupole might be accompanied by a large negative decapole error. This behavior is not accidental. The fractional gap width of the magnet is controlled to a mechanical tolerance which in our case was $\sim \pm 100 \mu$ out of a $50 \mathrm{~mm}$ gap or $\sim 0.2 \%$. The fluctuations in gap width allowed by this tolerance could be compensated by a Zshim which varied the magnet length by roughly the same $\sim 0.2 \%$ or $8 \mathrm{~mm}$ out of a $4 \mathrm{~m}$ magnet. In no case did we find a magnet with a field defect so extreme that it was impossible to define a set of shims which would bring it to zero. Within the constraints of custom shimming only one end of the magnet and a maximum $+/-1$ " shim length, we occasionally left one or more multipoles only partially corrected.

Skew multipoles can also be trimmed with this procedure, although less effectively. Early in the Recycler R\&D program we performed experiments to verify that skew multipoles could be trimmed as well. However it appeared from particle tracking studies that the skew multipoles observed in the magnets had no impact on the beam and were therefore ignored. The skew quadrupole was trimmed in these magnets by another technique, namely varying the relative strengths of the permanent magnet material behind the top and bottom pole tips.

A minor annoyance was the interaction between the strengths of the permanent magnets and the end shims. Magnets with strengths that had been previously trimmed to a few parts in $10^{-4}$ would find that changing a field quality shim would bring the magnet strength out of specifications. The strength trimming would then have to be repeated. The mechanism for this strength change can be understood by considering a sextupole shim, which redistributes flux towards or away from the center of the bore of the magnet and out towards the edges of the pole tips. The total flux available to the pole tip is determined by the magnetic material available. Thus a positive sextupole shim (longer at the edges of the aperture than at the center) will reduce the amount of flux seen by a probe (or the beam) located at the center of the aperture. In practice this was easily dealt with since the strength change was detected while the magnet was already mounted on the test stand and the amount of ferrite could be easily adjusted in situ.

\section{RESULTS}

A typical field error distribution before and after the full custom shims were applied is given in Fig. 4. In this case the RMS values of the octupole error were reduced by a factor of 4, and the systematic (average) value of the octupole was reduced by a factor of 5 . This was typical in cases where a full-custom shim was applied to each magnet. As mentioned previously, not all magnets required full custom shims in order to meet specifications. Thus the overall production distributions which included magnets with "semi-custom" shims was somewhat wider.

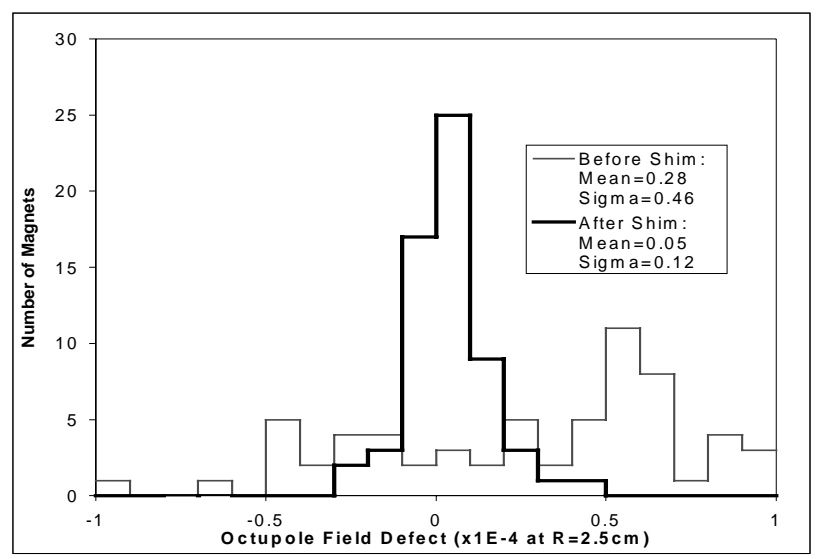

Fig. 4 - Effect of end shimming on the octupole error distribution for Recycler gradient magnets. The two curves show the octupole errors before and after fullcustom shims were applied. After shimming, random and systematic defects are at the $10^{-5}$ level $(\mathrm{R}=2.5 \mathrm{~cm})$

\section{CONCLUSIONS}

The technique of computer generated and machined custom end shims to cancel observed multipole errors proved a convenient and cost-effective method of improving the field quality of Recycler magnets. The technique described is generally applicable to warm-iron magnets and can be used to reduce at least the low multipoles errors to the limit of measurement accuracy or $\mathrm{dB} / \mathrm{B} \sim 10^{-5}$.

\section{REFERENCES}

[1] "Storage Ring for Increased Antiproton Production at Fermilab", G.W. Foster and G. P. Jackson, PAC '95. "Recycler Design Report", FNAL-TM-1991, G. P. Jackson, ed.

[2] "Simulation of the Recycler Ring Dynamic Aperture", D. Johnson and S. Mishra, PAC '99.

[3] "Temperature Considerations in the Design of a Permanent Magnet Storage Ring", K. Bertsche, G.W.Foster, and J-F. Ostiguy, PAC' 95.

[4] Most machine tools accept an ASCII code commonly referred to as "G-code" which is supposedly standardized but in practice differs significantly on different NC machines. My suggestion to anyone attempting this is to have your machinist make you a sample part, then ask him for a floppy disk file and code up something which produces modified versions of the code for the sample part. 